\section{Rapid mosaic brain evolution under artificial selection for relative telencephalon size in the guppy (Poecilia reticulata)}

\author{
Stephanie Fong ${ }^{1 *}$, Björn Rogell ${ }^{1,2}$, Mirjam Amcoff ${ }^{1}$, Alexander Kotrschal ${ }^{1,3}$, Wouter van der Bijl ${ }^{1,4}$, \\ Séverine D. Buechel ${ }^{1}$, Niclas Kolm ${ }^{1 *}$
}

The mosaic brain evolution hypothesis, stating that brain regions can evolve relatively independently during cognitive evolution, is an important idea to understand how brains evolve with potential implications even for human brain evolution. Here, we provide the first experimental evidence for this hypothesis through an artificial selection experiment in the guppy (Poecilia reticulata). After four generations of selection on relative telencephalon volume (relative to brain size), we found substantial changes in telencephalon size but no changes in other regions. Further comparisons revealed that up-selected lines had larger telencephalon, while down-selected lines had smaller telencephalon than wild Trinidadian populations. Our results support that independent evolutionary changes in specific brain regions through mosaic brain evolution can be important facilitators of cognitive evolution.

\section{INTRODUCTION}

The vertebrate brain contains a number of morphologically and functionally distinct regions, linked through intricate connective systems, ranging from short local links between adjacent structures to connections between spatially distant regions through myelinated axons. Although the vertebrate brain is predominantly conserved with regard to the organization of the regions it contains, there is large variation among vertebrate species in the relative size and function of different regions (1). One example is the disproportionately large neocortex in large-brained primates such as humans (2). However, how has such variation evolved among the vertebrates, and what are the functional consequences of this variation? Despite more than a century of research, these remain to be important questions in the study of brain evolution (3). One of the leading brain evolution theories, the mosaic brain evolution hypothesis (4), posits that changes in individual regions may occur due to region-specific selection (e.g., according to explicit cognitive or sensory requirements), relatively independent of changes in other regions. If true, then this should result in a heterogeneous pattern of brain evolution $(4,5)$. This is in contrast to the concerted brain evolution hypothesis, which states that brain regions evolve in a coordinated manner, possibly due to underlying developmental constraints $(2,6)$.

The mosaic brain evolution hypothesis has received ample support mainly from comparative analyses at the interspecific level by means of examining how ecological factors correlate with specific alterations in neural anatomy across species or populations, e.g., (7-12). One important example of empirical evidence for this hypothesis was provided by Barton and Harvey (4), where the authors analyzed the evolutionary changes in brain regions associated with specific functional units in primates and insectivores. The partly autonomous evolution of these functionally linked components with respect to other brain regions and overall brain size provided

${ }^{1}$ Department of Zoology, Stockholm University, Stockholm, Sweden. ${ }^{2}$ Department of Aquatic Resources, Swedish University of Agricultural Sciences, Drottningholm, Sweden. ${ }^{3}$ Department of Behavioural Ecology, Wageningen University, Wageningen, Netherlands. ${ }^{4}$ Department of Zoology, University of British Columbia, Vancouver, Canada. *Corresponding author. Email: steph.fongpf@gmail.com (S.F.); niclas.kolm@zoologi. su.se (N.K.) support for mosaic brain evolution in mammals. Despite a discernable bias toward mammalian studies, support for mosaic brain evolution in various fish and avian species has also been demonstrated (13). For example, three-dimensional reconstructions of the brains of African mormyrid fishes that use active electro sensing revealed an enlarged cerebellum in comparison to outgroup species without such a system (14). Another well-studied example concerns food caching in birds, where the ability to store food for later retrieval has been found to be positively correlated with hippocampus volume in species performing this cognitively demanding behavior (15-17).

Recently, intraspecific comparative analyses have complemented existing interspecific studies and shown positive correlations between song complexity and the brain regions governing singing behavior in song birds [HVC (high vocal center) and RA (robust nucleus of the archistriatum)] (18). Intraspecific comparisons provide the additional benefit of examining potential phenotypic plasticity effects brought about by different environmental conditions [e.g., (19)]. The potential for plastic changes in neural architecture is especially pronounced in teleost fish, given their prolonged neurogenesis throughout their entire lifetime [e.g., (19-21)]. That such plastic changes in specific brain regions occur in response to a multitude of factors supports the independent capacity of brain components.

Interspecific and intraspecific correlative comparative analyses form an important tool to investigate evolutionary patterns and generate hypotheses $(7,11,13)$. An experimental approach, on the other hand, enables the targeted modification of specific components while simultaneously allowing the determination of correlated changes in brain morphology, behavior, and physiology [see, for instance, (22)]. This is necessary to identify positive and negative genetic correlations, for instance, to reveal potential trade-offs with life history $(23,24)$. Experimental data are thus needed to understand the independent evolutionary potential of brain regions and its associated consequences. Artificial selection experiments on mice and fish have revealed that increases in relative brain size can occur quickly and yield important cognitive benefits [increased associative learning (24-29), more accurate mate preferences $(30,31)$, and more effective predator avoidance $(32,33)$ ] but also incur high energetic costs [lower offspring production (24), reduced innate immune response 
(34), and shorter intrinsic life span (35)]. However, in line with the mosaic brain hypothesis, relative brain size is a rather crude measure of brain morphology, and evolutionary changes in brain morphology in wild populations are unlikely to target the entire brain $(36,37)$. Another fitting and important aspect of the mosaic brain hypothesis concerns the costly nature of neural tissue, which should favor evolutionary expansion of only functionally relevant structures, thereby reducing unnecessary energy expenditure $(11,38)$. To increase our understanding of brain morphology evolution and to go a step beyond brain size (39), artificial selection experiments that target region evolution could be instrumental.

Here, we provide such an experimental test of the mosaic brain evolution hypothesis through an artificial selection experiment that targets relative telencephalon size (i.e., telencephalon volume in relation to the brain remainder) in guppies (Poecilia reticulata). The established role of the telencephalon as a cognitive center in fish $(40-42)$ and other vertebrates $(43,44)$ makes this region an appealing target of artificial selection with highly general implications, especially given the potential for future tests of the implicated costs and cognitive benefits. We chose the guppy as the model for the experiment because it is a live-bearing teleost species with a well-studied, interesting, and variable ecology, e.g., (45-48), it has a relatively short generation time (45), and large populations can be kept in the laboratory with ease. Specifically, we aimed to increase and decrease the relative size of the telencephalon in relation to the brain remainder while examining for concomitant changes in other aspects of brain morphology. We also investigated sex-specific effects. As a measure of potential reproductive costs (23) associated with changes in telencephalon size, offspring production, one of the best proxies for fitness in the guppy, was quantified during selection. Last, we investigated how the artificial selection lines compared to wild populations by comparing their relative telencephalon size against a large dataset spanning across 16 Trinidadian guppy populations.

\section{RESULTS}

After four generations of selection for relative telencephalon size (telencephalon volume in relation to the volume of the brain remainder), there was an average difference of $10.1 \%$ between the up-selected and down-selected females and an average difference of $9.5 \%$ in males. There was an overall significant difference between selection treatments for both females and males [estimated differences between up-selected and down-selected lines: $\beta$, presented with $95 \%$ credible intervals (CI); females: $\beta=0.046$ [0.0094; 0.089], $P_{\text {MCMC }}=0.044 ;$ males: $\beta=0.022$ [0.0052; 0.041], $\left.P_{\text {MCMC }}=0.028\right]$ (Fig. 1 and Table 1). Comparisons to the unselected control lines revealed nonsignificant trends for selection to proceed in an asymmetrical manner in females (up-selected versus control line: $\beta=0.031$ $[-0.0023 ; 0.065], P_{\mathrm{MCMC}}=0.052$; down-selected versus control line: $\left.B=-0.014[-0.077 ; 0.064], P_{\mathrm{MCMC}}=0.50\right)$, but no such pattern was detected in males (up-selected versus control line: $\beta=0.030[-0.017$; $0.098], P_{\mathrm{MCMC}}=0.18$; down-selected versus control line: $B=-0.0094$
A

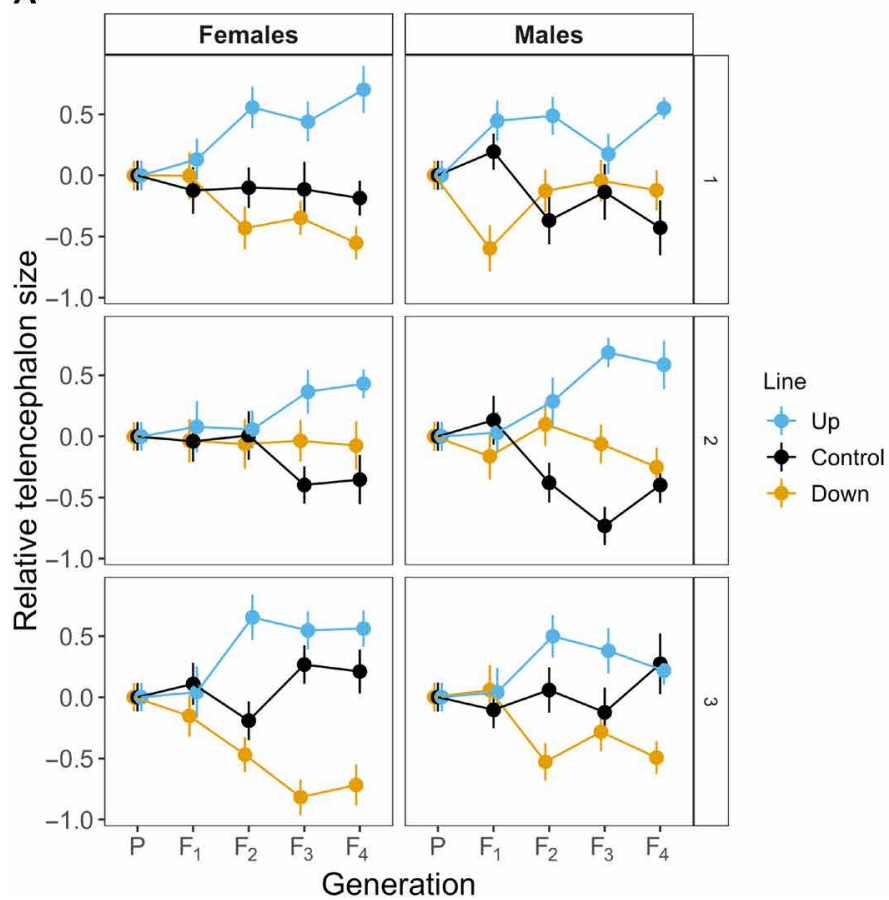

B

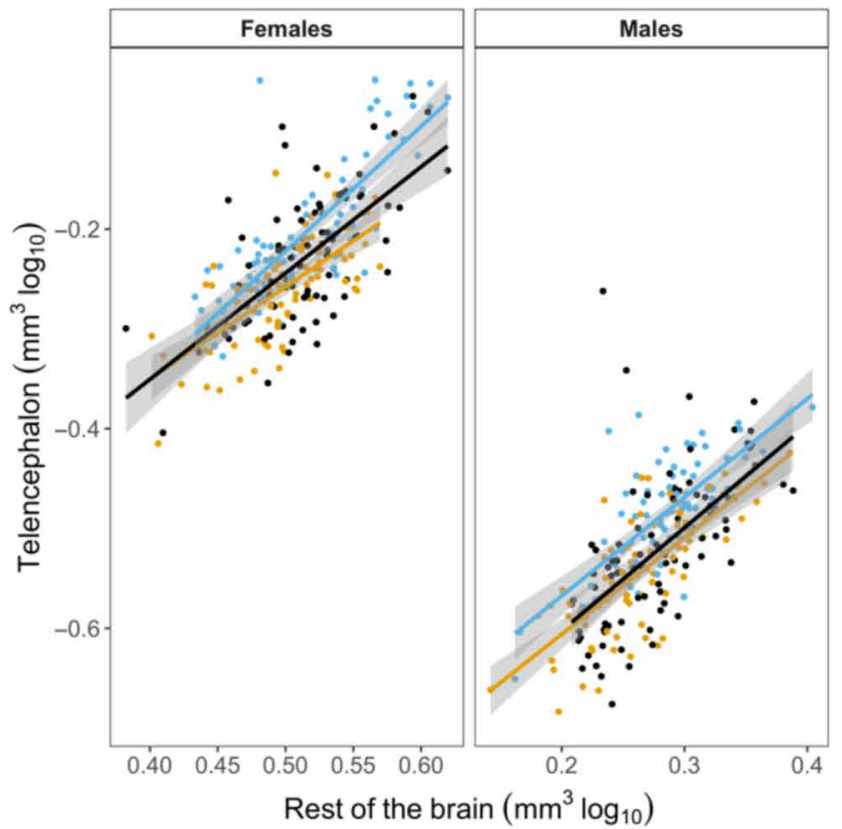

Fig. 1. Response to artificial selection on relative telencephalon size in both females and males. (A) Relative telencephalon size in response to directional selection over four generations. P indicates the starting parental population. Depicted are the means and SE values (SEM) for standardized residuals of telencephalon volume regressed on the volume of the brain remainder (total brain volume minus telencephalon volume) within each generation and replicate. The figure shows the selection response across the three replicates, with the left and right panels separately illustrating the response in females and males, respectively. (B) Overall change in telencephalon size in generation $\mathrm{F}_{4}$ females and males. Plotted on the $y$ axis are the raw data of telencephalon volume (log-transformed), with brain remainder (log-transformed) on the $x$ axis. Different selection treatments are indicated by the different colored lines and points (down-selected line, yellow; unselected control line, black; and up-selected line, blue). 
Table 1. Model output for relative telencephalon size (relative to the remainder of the brain) in generation $F_{4}$ females and males, presented with mean estimates and $95 \% \mathrm{Cl}$. The intercept is set to the intercept of the regression of log telencephalon size on log brain remainder (total brain volume minus telencephalon volume) for the down-selected line in females. Mean estimates are indicative of differences between the intercept (down-selected females) and the specified variable (conforming to the default contrast matrix in R). NA, not applicable. ${ }^{* *} P<0.001,{ }^{* *} P<0.01,{ }^{*} P<0.05$.

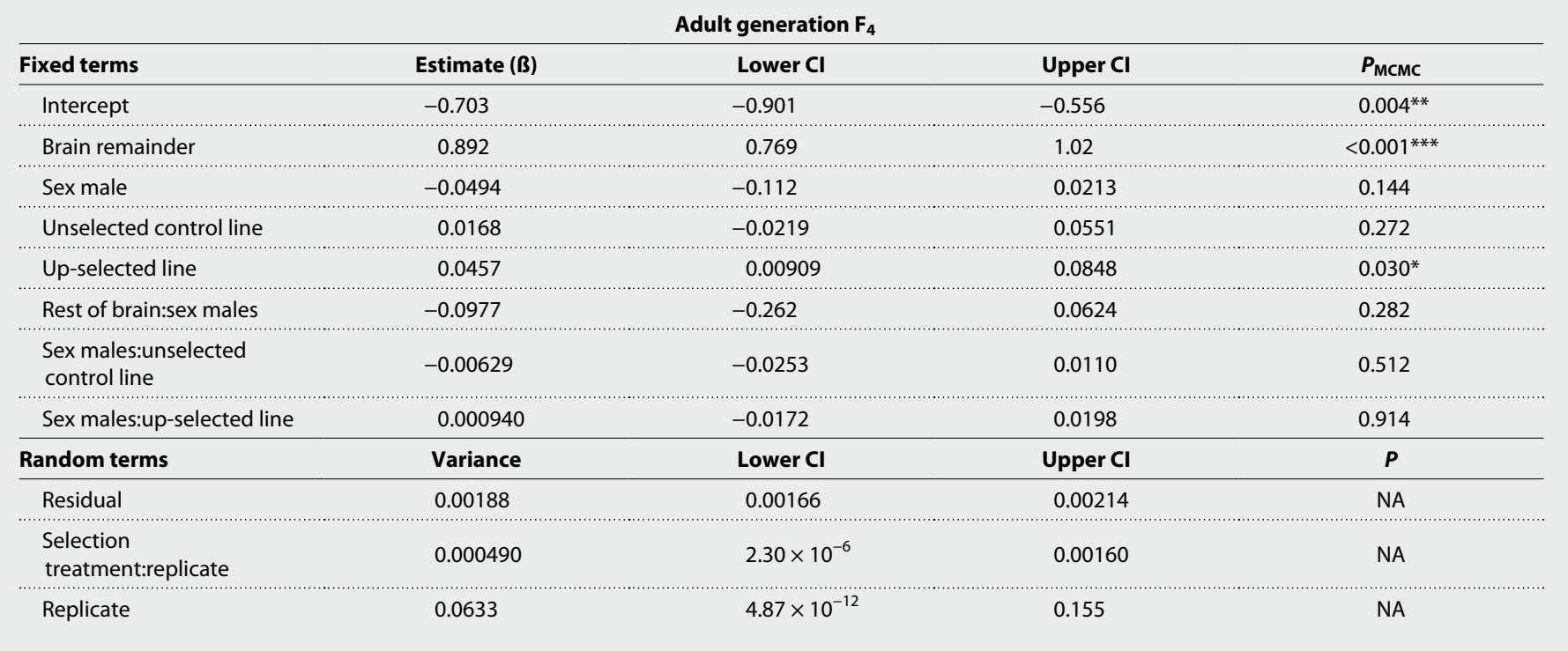

$\left.[-0.064 ; 0.050], P_{\text {MCMC }}=0.66\right)$. The effects of selection were consistent also when controlling for body length (table S1), suggesting that relative telencephalon size, both in relation to the brain remainder and body size, was affected by the directional selection procedure.

Results presented hereafter are based on comparisons between down-selected and up-selected lines only, unless otherwise stated (for a full summary of results, see Table 2, table S2, and figs. S1 and S2). No significant differences in any of the other brain regions assessed were detected (Table 2, table S2, and figs. S1 and S2). However, brain size (controlling for body length) was found to be larger in the up-selected lines $\left(~ B=0.023[0.0073 ; 0.042], P_{\text {MCMC }}=0.022\right)$. Further analysis across each sex revealed that this difference in relative brain size was mainly driven by effects in males (males: $\beta=0.022$ [0.0052; 0.041], $P_{\mathrm{MCMC}}=0.028$; females: $\beta=0.025$ $\left.[-0.0070 ; 0.058], P_{\mathrm{MCMC}}=0.098\right)$ (table S3). Exclusion of the telencephalon (total brain volume minus telencephalon volume) showed a nonsignificant trend for a difference between selection treatments ( $ß=0.017[-0.0023 ; 0.036], P=0.074)$. Together with the lack of difference in any of the other brain regions, this supports that the trends toward larger overall brain size in the up-selected male lines were mainly the result of the significantly larger telencephalon in these lines. Realized heritabilities of relative telencephalon size were variable but congruent between sexes when comparing the up- and downselected lines against the control lines (up-selected females: 1.03 [-0.66; 4.35], down-selected females: 0.44 [-1.37; 2.73], up-selected males: 1.42 [-1.78; 7.00], and down-selected males: 0.0078 [-1.92; 1.50]).

A previous study comparing the brain morphology of wild-caught and laboratory-reared guppies found that the latter had smaller telencephalon and optic tectum in relation to the former after just a single generation (49). Here, we compared the mean telencephalon size between selection treatments and 16 Trinidadian wild populations in females and found that the unselected control lines had a telencephalon size that most closely resembled that found in natural populations, while up-selected and down-selected lines had larger and smaller relative telencephalon size than natural populations (Fig. 2). Upon closer examination, it was also found that selection over four generations resulted in a comparable range of relative telencephalon sizes in our selected sample (range, -0.76 to 1.3 ) in comparison to natural populations (range, -1.2 to 0.67 ), contradicting inbreeding issues in this trait over several generations of selection in our experiment (fig. S3).

As there is a strong correlation between female body size and reproductive output, we first tested for any difference in standard length across selection treatments but found no such effect (upselected versus down-selected: $\beta=0.0070[-0.019 ; 0.036], P_{\mathrm{MCMC}}=$ $0.50)$. The number of offspring produced during the first parturition was thereafter compared across selection treatments to assess for potential reproductive costs implicated in the selection for a larger telencephalon. There was no difference in the number of offspring produced across selection treatments (up-selected versus downselected: $\beta=0.19[-0.21 ; 0.59], P_{\text {MCMC }}=0.28$; Fig. 3$)$.

\section{DISCUSSION}

Our results show that the relative size of a brain region can evolve independently and quickly under strong directional selection. Hence, our results provide experimental support for the mosaic brain evolution hypothesis $(4,5)$. This means that brain regions can display rapid and largely independent evolutionary changes, which further imply that selection can alter individual brain regions in response to directed selection pressures, one of which could be specific cognitive demands imposed by the environment. The rate of evolution shown here is similar to that seen when brain size was the target of selection (24); a 9\% difference in brain mass between the large- and small-brain lines was found after two generations of artificial selection and a $15 \%$ difference after five generations of selection (50). 
Table 2. Model output for overall changes in brain morphology in generation $F_{4}$ adults in response to selection for relative telencephalon size, presented with mean estimates and $\mathbf{9 5 \%} \mathrm{Cl}$. The intercept is set to the intercept of the regression of log brain region size on log brain remainder (brain volume minus volume of region of interest) for the down-selected line in females. The mean values presented in the table represent differences between the intercept (down-selected females) and the specified variable (conforming to the default contrast matrix in $\mathrm{R}$ ). ${ }^{* * *} P<0.001,{ }^{* *} P<0.01,{ }^{*} P<0.05$.

\begin{tabular}{|c|c|c|c|c|}
\hline & Estimate (ß) & Lower CI & Upper Cl & $P_{\text {MCMC }}$ \\
\hline \multicolumn{5}{|l|}{ Overall brain } \\
\hline Intercept & -0.677 & -0.898 & -0.445 & $0.002^{* *}$ \\
\hline Standard length & 0.897 & 0.776 & 1.02 & $<0.001^{* * *}$ \\
\hline Sex male & -0.364 & -0.598 & -0.126 & $0.004^{* *}$ \\
\hline Unselected control & 0.00756 & -0.00914 & 0.0260 & 0.278 \\
\hline Up-selected line & 0.0233 & 0.00730 & 0.0423 & $0.022^{*}$ \\
\hline Standard length:sex male & 0.232 & 0.0357 & 0.408 & $0.016^{*}$ \\
\hline Sex male:unselected control & 0.000502 & -0.0110 & 0.0129 & 0.926 \\
\hline Sex male:up-selected line & -0.00136 & -0.0139 & 0.0107 & 0.842 \\
\hline \multicolumn{5}{|l|}{ Optic tectum } \\
\hline Intercept & 0.00619 & -0.0108 & 0.0701 & 0.138 \\
\hline Rest of brain & 0.656 & 0.575 & 0.737 & $<0.001^{* * *}$ \\
\hline Sex male & -0.0251 & -0.00217 & 0.0491 & 0.076 \\
\hline Unselected control & -0.00141 & -0.0139 & 0.0162 & 0.846 \\
\hline Up-selected line & -0.00606 & -0.0225 & 0.00969 & 0.370 \\
\hline Rest of brain:sex male & -0.137 & -0.246 & -0.0256 & $0.010^{* *}$ \\
\hline Sex:unselected control & 0.00254 & -0.0104 & 0.0168 & 0.676 \\
\hline Sex:up-selected line & -0.00488 & -0.0191 & 0.00988 & 0.518 \\
\hline \multicolumn{5}{|l|}{ Cerebellum } \\
\hline Intercept & -0.740 & -0.858 & -0.548 & $<0.001^{* * *}$ \\
\hline Rest of brain & 1.13 & 0.970 & 1.29 & $<0.001^{* * *}$ \\
\hline Sex male & -0.208 & -0.294 & -0.131 & $<0.001^{* * *}$ \\
\hline Unselected control & 0.00678 & -0.0625 & 0.0539 & 0.758 \\
\hline Up-selected line & -0.00500 & -0.0625 & 0.0543 & 0.790 \\
\hline Rest of brain:sex male & 0.264 & 0.0756 & 0.492 & $0.010^{*}$ \\
\hline Sex:unselected control & -0.0116 & -0.0326 & 0.00984 & 0.292 \\
\hline Sex:up-selected line & 0.00844 & -0.0118 & 0.0316 & 0.456 \\
\hline \multicolumn{5}{|l|}{ Dorsal medulla } \\
\hline Intercept & -0.725 & -1.03 & -0.454 & $0.010^{* *}$ \\
\hline Rest of brain & 0.628 & 0.416 & 0.817 & $<0.001^{* * *}$ \\
\hline Sex male & -0.153 & -0.262 & -0.0368 & $0.014^{*}$ \\
\hline Unselected control & -0.00234 & -0.0423 & 0.0333 & 0.854 \\
\hline Up-selected line & 0.00582 & -0.0331 & 0.0456 & 0.700 \\
\hline Rest of brain:sex male & 0.143 & -0.118 & 0.404 & 0.312 \\
\hline Sex:unselected control & 0.00329 & -0.0236 & 0.0324 & 0.842 \\
\hline Sex:up-selected line & 0.00769 & -0.0217 & 0.0361 & 0.654 \\
\hline \multicolumn{5}{|l|}{ Hypothalamus } \\
\hline Intercept & -0.714 & -0.834 & -0.610 & $0.008^{* *}$ \\
\hline Rest of brain & 0.577 & 0.423 & 0.751 & $<0.001^{* * *}$ \\
\hline Sex male & -0.0413 & -0.132 & 0.0529 & 0.396 \\
\hline Unselected control & 0.00802 & -0.0191 & 0.0395 & 0.542 \\
\hline Up-selected line & 0.0110 & -0.0163 & 0.0408 & 0.442 \\
\hline
\end{tabular}

continued on next page 


\begin{tabular}{|c|c|c|c|c|}
\hline & Estimate ( $(B)$ & Lower $\mathrm{Cl}$ & Upper CI & $P_{\text {MCMC }}$ \\
\hline Rest of brain:sex male & -0.291 & -0.517 & -0.0966 & $0.006^{* *}$ \\
\hline Sex:unselected control & 0.00740 & -0.0142 & 0.0312 & 0.651 \\
\hline Sex:up-selected line & 0.0216 & -0.00224 & 0.0449 & 0.074 \\
\hline \multicolumn{5}{|l|}{ Olfactory bulbs } \\
\hline Intercept & -2.27 & -2.73 & -1.88 & $<0.001^{* * *}$ \\
\hline Rest of brain & 1.02 & 0.657 & 1.34 & $<0.001^{* * *}$ \\
\hline Sex male & -0.0580 & -0.280 & 0.144 & 0.600 \\
\hline Unselected control & -0.0170 & -0.0846 & 0.0504 & 0.514 \\
\hline Up-selected line & 0.0290 & -0.0440 & 0.103 & 0.312 \\
\hline Rest of brain:sex male & -0.202 & -0.668 & 0.227 & 0.386 \\
\hline Sex:unselected control & 0.0640 & 0.0165 & 0.110 & $0.006^{* *}$ \\
\hline Sex:up-selected line & -0.0149 & -0.0639 & 0.0307 & 0.542 \\
\hline
\end{tabular}

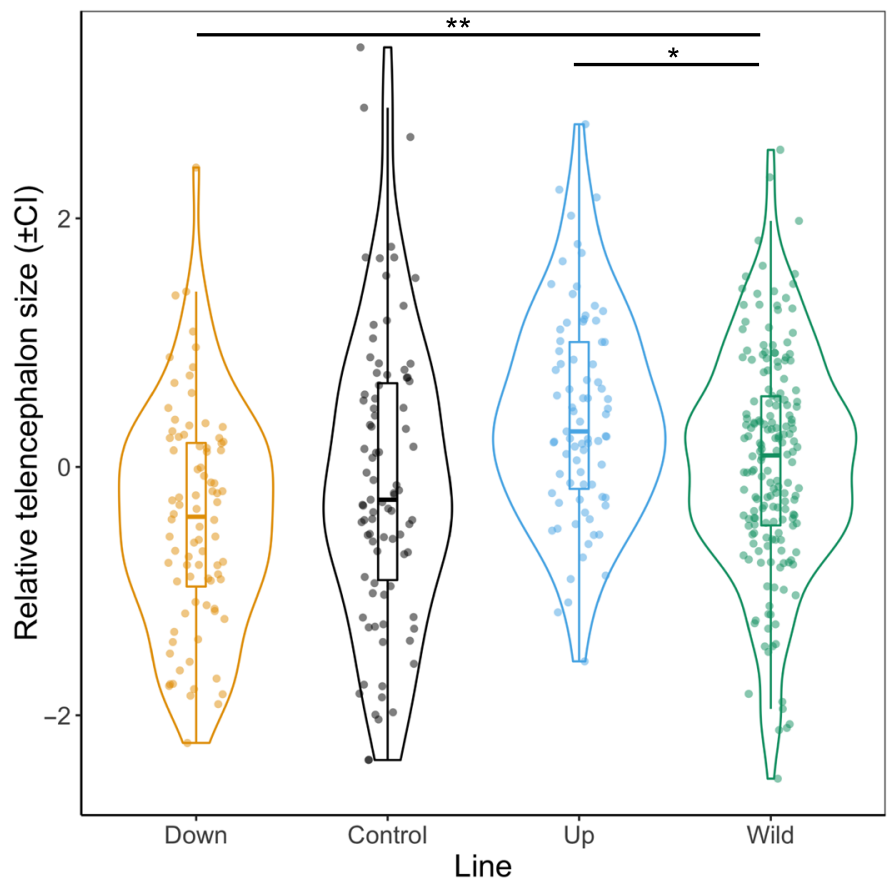

Fig. 2. Female relative telencephalon size after three rounds of selection (generation $\mathrm{F}_{4}$ ) in comparison to wild fish obtained from 16 wild populations in Trinidad. Plotted on the $y$ axis are the estimated marginal means and $95 \%$ confidence intervals, obtained from standardized residuals of telencephalon volume regressed on brain remainder (total brain volume minus telencephalon volume). Pairwise comparisons were performed with respect to the wild populations, and $P$ values were calculated following Holm's adjustment for multiple comparisons. ${ }^{* *} P<0.01 ;{ }^{*} P<0.05$.

That such rapid changes in brain morphology, both in terms of overall brain size and brain regions, can occur in response to directional selection is consistent with the assumed high evolvability of the brain (51).

In contrast to the aforementioned artificial selection experiment on relative brain size in guppies, which found a clear reduction in fecundity (i.e., lower offspring number) in the large-brain lines (24), we did not find any evidence for any reproductive trade-off with

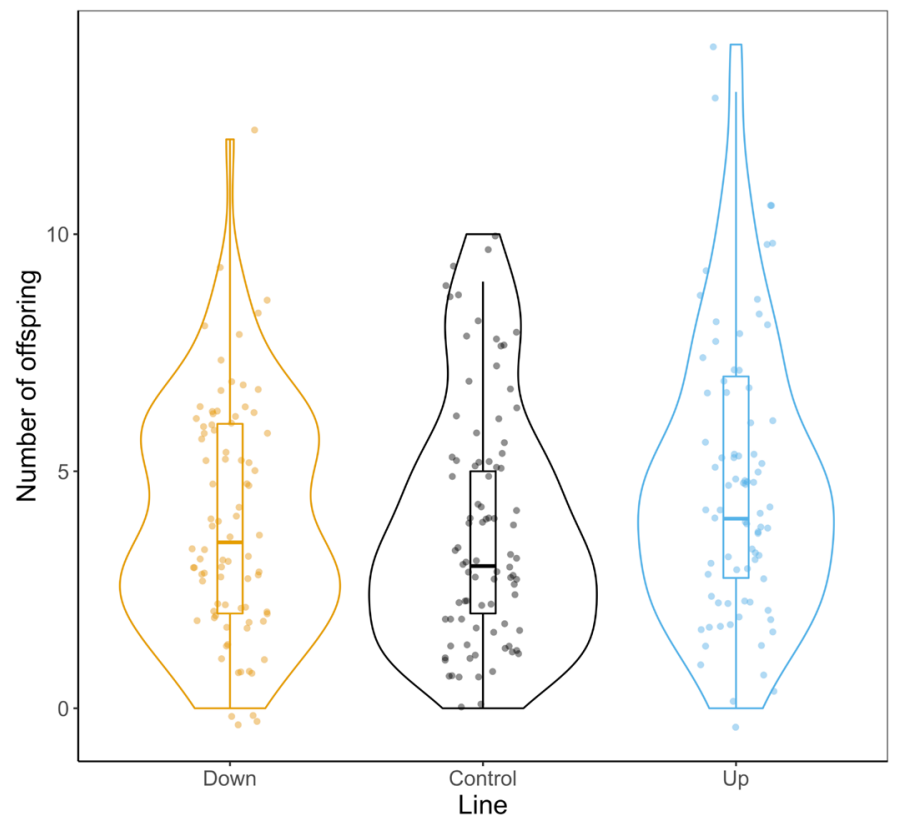

Fig. 3. Reproductive output, as assessed by the number of juveniles produced during the first parturition, over the different selection lines. Error bars represent SEM. No significant differences were detected in generation $\mathrm{F}_{4}(P>0.2)$.

telencephalon size. Together, these results indicate that mosaic brain evolution of key regions associated to cognitive demands may be an energy-effective and thus potentially favored way to reduce life history costs during cognitive evolution. As mentioned previously, the comparative and experimental evidence for the costs associated with increased brain size is substantial. However, little is known about the potential costs associated with variation in brain region size. A potential additional route for future research is therefore to investigate whether life history costs are associated also with vertebrate brain region variation in comparative analyses.

We found no evidence for strong associations in the size between different brain regions. Only the individual region targeted by our selection regime, the telencephalon, changed significantly in size 
during the experiment. This matches a previous study examining phenotypic and genotypic correlations between brain regions in threespined stickleback (Gasterosteus aculeatus), where Noreikiene et al. (52) found that brain regions shared relatively low genetic correlations. Moreover, in a large-scale quantitative genetic analysis study comprising approximately 10,000 mice, different brain regions were found to be regulated by distinct nonoverlapping loci, allowing for the selection of individual brain components (53). The lack of a link between telencephalon size and other brain regions also speaks against prominent trade-offs between investments into separate regions. This is somewhat unexpected, given that neural tissue is one of the most energetically costly tissues $(24,34,35,54)$. Our experimental design, which targeted relative telencephalon size in relation to the brain remainder, should also have been effective in revealing potential developmental trade-offs with other brain regions. Again, the lack of evidence for any trade-off in this context may stem from high independence and the low genetic correlations between the sizes of different brain regions. Although our setup was designed to select for telencephalon volume in relation to brain remainder, another potentially important predictor of cognitive ability is the relative size of the telencephalon in relation to body size. It is therefore worth noting that the difference in telencephalon size between selection treatments was significant in relation to both brain remainder and body size, demonstrating the robustness of the selection procedure and generality of our results. Note that our animals were kept under benign laboratory conditions, which may have masked potential energetic trade-offs. To fully understand the intricate evolutionary associations between different brain regions will require more work (e.g., rearing of individuals under different food restriction treatments to mimic natural conditions), ideally also into the interconnectivity between regions (13). The increased telencephalon size in the up-selected lines led to an increase in relative brain size but mainly in males (the trend, albeit not statistically significant, was in the same direction also in females; fig. S2 and table S2). Substantial changes in the size of specific regions must of course affect also the overall brain size unless there are trade-offs with other regions (14). However, comparative analyses have demonstrated that changes in some regions, especially neocortex size and cerebellum size, are linked to changes in brain size $[$ e.g., $(3,6,14)]$. Although the effect was mostly evident in males after four generations of selection and apparently mainly driven by the larger telencephalon in these lines, our results provide support for a similar pattern also at the intraspecific level. While more work is clearly needed to unveil the developmental pathways and functional implications of the link between telencephalon size and brain size [e.g., (55)], we propose that mosaic brain evolution can be an important first step also toward larger brain size.

Comparative studies often find support for a combination of mosaic and concerted evolution $(34,52,53)$. How do such patterns go together with our experimental results? Artificial selection experiments reveal what is possible under strong directional selection and do not necessarily reflect exactly the patterns and processes that are occurring in wild populations. Hence, while our experiment shows that telencephalon size can evolve highly independently, many opposing selection pressures such as brain cavity space constraints (56) and/or developmental and energetic constraints $(2,6,57)$ may impede the signatures of selection for mosaic brain evolution in wild populations. Selection on cognitive ability in wild populations may also target other behavioral and morphological aspects than brain region size (58). One possible reason why we found it harder to decrease, rather than increase, the size of the telencephalon could be limitations due to cognitive thresholds, whereby a minimum size requirement of the telencephalon is necessary. As mentioned earlier, the teleost telencephalon has known implications in a wide range of cognitive processes, including, but not limited to, spatial learning and fear conditioning response. It is therefore conceivable that a fundamental size is required for normal daily functioning, hence curbing the extent to which down-selection can progress. This is in accordance with Haller's rule, whereby smaller animals tend to have relatively larger brains due to the minimum absolute brain size required for performing basic cognitive functions (59).

Realized heritabilities in relative telencephalon size were substantial at least in the up-selected treatments in both sexes but showed high variation. This implies that changes in telencephalon size rests on an important heritable genetic background. While heritability in overall brain volume has been found to be high across species [e.g., humans $(60,61)$, rhesus monkeys (62), and baboons (63)], genetic influences on brain region sizes have been shown to vary considerably (63-66). In the previous artificial selection on brain size in guppies, realized heritability of relative brain size was found to be relatively high (24), suggesting that overall brain size is under strong genetic control. This is congruent with results from human studies where brain volume is substantially influenced by genetic factors, while heritability of individual brain components may vary throughout the life span (60). The measure of heritability used here estimates the proportion of total variance that can be attributed to genetic effects (67). The fact that we found evidence for considerably higher heritability estimates in the up-selected lines could once again be related to the aforementioned cognitive threshold theory, whereby the requirement for a minimum telencephalon size outweighs genetic influences. Although substantial, the values of realized heritability presented here should be interpreted with some caution. For instance, that the values of realized heritability sometimes lay outside of the normal range of 0 to 1 may implicate effects of phenotypic plasticity. Given that both brain size and brain region sizes are highly plastic, it is possible that environmental effects may result in a range of region sizes outside of the norm. In addition, it is worth noting that measurement error is generally larger for brain regions than, for instance, for overall brain size.

Last, our comparison of the telencephalon selection lines in relation to 16 recently sampled wild populations of guppies shows that the control lines are most similar to the wild populations, while up-selected and down-selected lines have larger and smaller telencephali than the wild populations, respectively. Although telencephalon size in our selection lines mostly falls within the range of the wild populations, telencephalon size in one specific replicate of the up-selected line was larger in comparison to the natural range. These results are consistent with the following: (i) Ample genetic variation still exists in the wild-type laboratory strain of guppies used as the basis for this selection experiment $(68,69)$, and (ii) the selection procedure used here has mainly acted within the natural range of telencephalon size variation. Further selection for additional generations will be undertaken to test whether artificial selection on a separate brain region can shift its phenotype even further beyond the naturally occurring levels, similar to what has been done for other morphological traits on numerous occasions (70-73).

In conclusion, our results show that brain morphology can evolve rapidly in a highly independent fashion under strong directional 
selection. Our study thus provides experimental support for the mosaic brain evolution hypothesis at the intraspecific level, and we propose that mosaic evolution can be an important facilitator of cognitive evolution at the intraspecific level.

\section{MATERIALS AND METHODS}

\section{Artificial selection for relative telencephalon size}

We selected on telencephalon size relative to the size of the rest of the brain, because the alternative, to select on relative telencephalon size against body size, was likely to create larger and smaller relative brain size due to the strong positive correlation between telencephalon size and brain size in vertebrates (74). Guppies ( $P$. reticulata) originating from a high-predation population in Trinidad and subsequently kept in large populations in the laboratory since 1998 were used as breeding stock for the starting population. The fish that formed the base populations for these selection lines were housed in large mixed sex groups in three 200-liter aquaria. One hundred and twenty breeding pairs were netted out and housed in 3-liter aquaria with light-colored gravel, constant aeration, freely floating live or plastic plants, and $>4$ water snails (Planorbis sp.) to consume organic waste. The laboratory was maintained at $25^{\circ} \pm 2^{\circ} \mathrm{C}$ with a 12 -hour light, 12-hour dark schedule. Fish were fed 6 days a week with a diet of flake food and freshly hatched brine shrimp. Given the cannibalistic nature of female guppies, a plastic net with mesh measuring $0.4 \mathrm{~cm}$ by $0.4 \mathrm{~cm}$ was placed at the front of the tank, functioning as a safe zone for fry while restricting the access of adult females. Tanks were scanned once every other day for any newborn offspring, which were removed and placed in similar tanks and kept to a maximum of six individuals per tank. Among these offspring, males were separated from females at the first sign of sexual maturity (i.e., visual detection of gonopodium development) and housed in groups of three to four individuals in similar 3-liter aquaria.

At $140 \pm 7$ (means \pm SD) days of age, 450 of these fish were used to establish the starting population $\left(\mathrm{F}_{0}\right)$ of three independent experimental replicates of 75 breeding pairs each in similar tanks as described above. Since quantification of brain morphology could not be performed in live fish, we allowed the pairs to produce at least two clutches or at least 12 juveniles per pair, before being euthanized for brain morphology quantification. Breeding pairs were euthanized in a water bath containing an overdose of benzocaine $(0.4 \mathrm{~g} /$ liter $)$ and fixed with $4 \%$ formalin in buffered phosphate-buffered saline (PBS) for 7 days. The samples were then washed twice in PBS and stored at $4^{\circ} \mathrm{C}$ awaiting dissection.

Standard length of all fish was recorded to the nearest $0.01 \mathrm{~mm}$, measured from the tip of the snout to the end of the caudal peduncle using digital calipers. Whole brains were dissected out from each fish under a dissecting microscope (Leica MZFLIII), and each individual brain was photographed from four separate views with a digital camera (Leica DFC 490). On the basis of these separate images, the length, width, and height of six major brain regions (i.e., telencephalon, optic tectum, cerebellum, dorsal medulla, hypothalamus, and olfactory bulbs) were measured using ImageJ software (fig. S4) (75). The volume of each of these brain regions was then determined using the ellipsoid model $(76,77)$

$$
V=(L \times W \times H) \frac{\pi}{6}
$$

Correspondingly, total brain volume was derived as the sum of the six measured brain regions. To select for relative telencephalon size, we first extracted the residuals from sex-specific regressions of telencephalon volume on the volume of the brain remainder excluding the telencephalon and standardized these to a mean of 0 and an SD of 1 . On the basis of the sum of the standardized residuals of each pair, offspring from the top and bottom 15 pairs (i.e., top and bottom $20 \%$ ) were chosen to form the next generation $\left(F_{1}\right)$ of upselected and down-selected lines, respectively, across three replicates. Additional offspring from 15 random pairs $\left(\mathrm{F}_{0}\right)$ were likewise used to form the control lines (see Fig. 4 for a detailed schematic outline of the selection procedure). The procedure was largely adapted from a previously successful artificial selection experiment for relative brain size in guppies (23). Each experimental replicate and selection

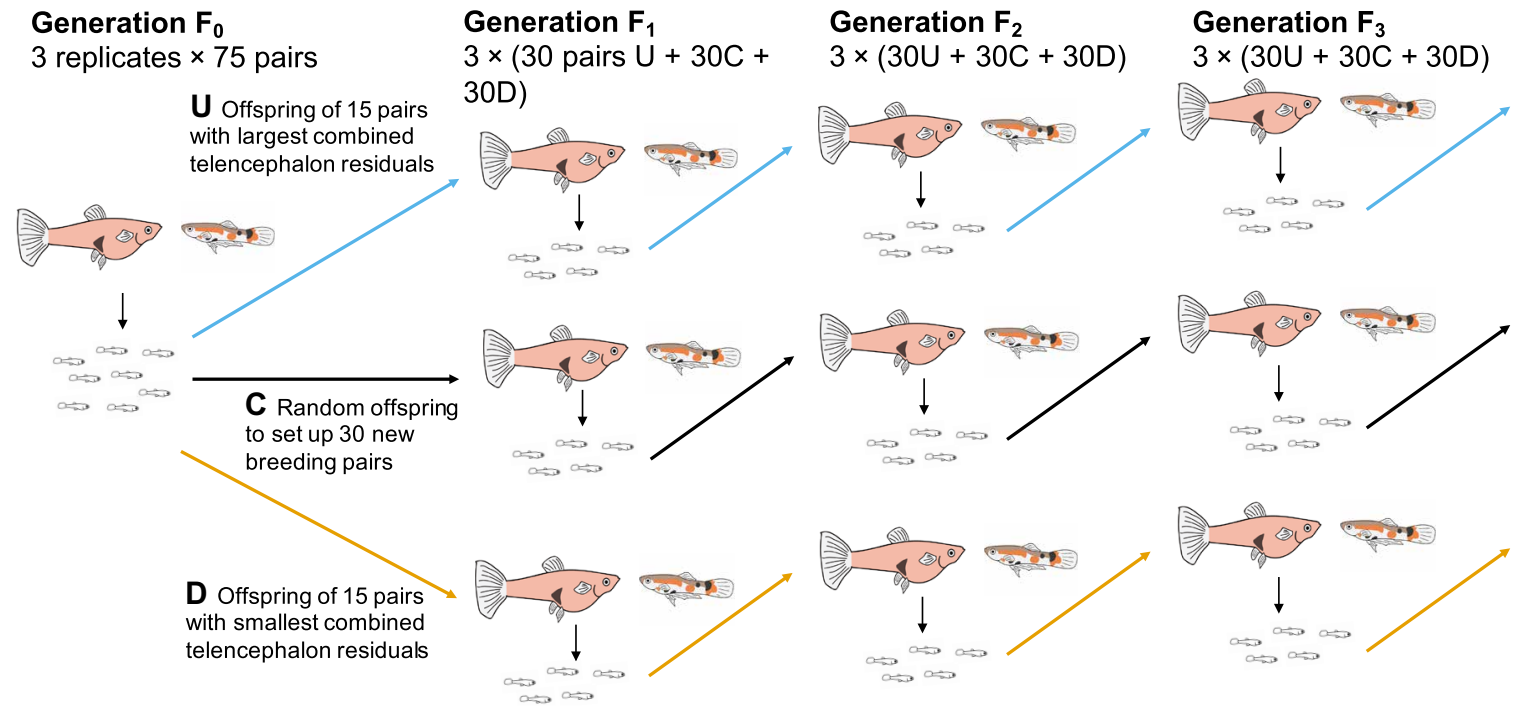

Fig. 4. Artificial selection procedure for relative telencephalon size. Different selection lines are indicated by the different colored arrows [i.e., blue for up-selection (U), black for control line (C), and orange for down-selected (D)] within each generation, and three independent replicates of 30 breeding pairs for each selection line were set up. 
regime combination was treated independently, thus giving a total of nine distinct populations (three replicates of up-selected, downselected, and control lines, respectively). Within each of the selection lines for each replicate, two females and two males from each of the selected $\mathrm{F}_{0}$ pairs were used to form 30 breeding pairs for the next generation $\left(\mathrm{F}_{1}\right)$, resulting in a total of 270 pairs. Again, the top and bottom 15 pairs in terms of relative telencephalon size were chosen for up-selected and down-selected lines, while the control lines were paired randomly in each of the following generations. To prevent inbreeding, breeding pairs never consisted of full siblings.

Similar to the previous generation, $\mathrm{F}_{1}$ breeding pairs were allowed to produce at least two clutches or at least 12 juveniles before whole brains were dissected out and measured. The selection proceeded in an identical manner as generation $\mathrm{F}_{0}$, first by ranking the parents according to the sum of parental residuals and using the respective offspring to propagate the subsequent generations $\left(\mathrm{F}_{2}, \mathrm{~F}_{3}\right.$, and $\left.\mathrm{F}_{4}\right)$.

\section{Brain morphology of wild fish populations}

To compare the brain morphology of the telencephalon selection lines to that of wild fish, the brain morphology of wild indigenous female guppies $(n=187)$ that were obtained from 16 different sites in Trinidad (36) was quantified in an identical manner as described above. These measurements could thereafter be used as a reference to assess the rate of brain evolution in our selection lines in relation to wild populations.

\section{Reproductive costs}

The development and maintenance of neural tissue incur high energetic costs $(54,78)$. To examine possible reproductive trade-offs involved in evolving a larger telencephalon, we assessed the possible costs in terms of reproductive output across selection lines. For this, we recorded the total number of juveniles and broods produced by all breeding pairs as a measure of reproductive success.

\section{Statistical analysis}

All statistical analyses were conducted in R statistical software v3.5.1 (79). We adopted a Bayesian approach in the R package MCMCglmm (80) to analyze for selection response/effects across lines, with flat priors for the fixed effects and locally uninformative priors for the random effects. We ran each model with $1.65 \times 10^{6}$ iterations, with a thinning interval of 1500 and a burnin of $1.5 \times 10^{5}$, resulting in an effective sample size of at least 1000 . All autocorrelations were within the range of -0.1 to 0.1 and were hence deemed acceptable. To avoid statistical confounds resulting from the inclusion of the region being analyzed, we fitted telencephalon volume (log-transformed) as the response variable against the brain remainder excluding the telencephalon (also log-transformed) as the covariate. Our model consisted of the following fixed effects: selection treatment (downselected, control line, and up-selected), brain remainder, and sex, with two-way interactions between brain remainder and sex and between sex and selection treatment. Replicate and the interaction between selection treatment and replicate were included as random factors. The intercept term was set to the regression of telencephalon size on brain remainder for females in the down-selected line.

To test whether selection results stemmed from an indirect selection on overall brain size, we fitted a similar MCMCglmm model with standard length, sex, selection treatment, and two-way interactions between standard length and sex and between sex and selection treatment as fixed effects. Random factors included replicate and the interaction between selection treatment and replicate. Analogous models were used to examine for potential correlated selection between the size of telencephalon and other brain regions in each of the five other unselected regions, i.e., optic tectum, cerebellum, dorsal medulla, hypothalamus, and olfactory bulbs (table S4).

Realized heritability was estimated as the ratio between the mean response to selection and the mean selection differential for each generation, for "up" and "down" selected lines (in comparison to the control lines), and males and females separately. We ran separate models for each generation and sex combination to estimate the response to selection (calculated as the difference between the up or down lines as compared to the control lines). For the models estimating the selection differential from the means of the selected individuals and the population mean (the mean of the guppies in the selection regimes for either smaller or larger telencephalon), we ran models specific to each generation and sex combination. The models estimating the response to selection contained the explanatory variables selection (up or down and "control") and log brain remainder (total brain volume minus volume of the telencephalon), with replicate and interaction between selection treatment and replicate as random effects. The models estimating the means of the selected individuals contained the explanatory variables selected ("yes" or "no"), log brain remainder as a covariate, and replicate line as random effect. To evaluate main effects independently of the covariate, $\log$ brain remainder was standardized to a mean of 0 and an $\mathrm{SD}$ of 1 . All models were fit using MCMCglmm as previously described. The full posterior distribution was included in all analysis.

Trade-offs, in terms of reproductive output, were analyzed by comparing the number of offspring produced during the first parturition for generation $\mathrm{F}_{4}$. Since female body size is known to be correlated with fecundity (81), standard length was included as a covariate in the model. Given the distribution of our response variable (i.e., number of offspring), we fitted a Bayesian model with a Poisson distribution. Hence, our model consisted of the following fixed effects: standard length, selection treatment, and the interaction between the two. Random factors included replicate and the interaction between selection treatment and replicate.

\section{SUPPLEMENTARY MATERIALS}

Supplementary material for this article is available at https://science.org/doi/10.1126/ sciadv.abj4314

View/request a protocol for this paper from Bio-protocol.

\section{REFERENCES AND NOTES}

1. R. G. Northcutt, Understanding vertebrate brain evolution. Integr. Comp. Biol. 42, 743-756 (2002).

2. B. L. Finlay, R. B. Darlington, Linked regularities in the development and evolution of mammalian brains. Science 268, 1578-1584 (1995).

3. G. F. Striedter, R. G. Northcutt, Brains Through Time: A Natural History of Vertebrates (Oxford Univ. Press, 2019).

4. R. A. Barton, P. H. Harvey, Mosaic evolution of brain structure in mammals. Nature $\mathbf{4 0 5}$, 1055-1058 (2000).

5. G. F. Striedter, Principles of Brain Evolution (Sinauer Associates, 2005).

6. B. L. Finlay, R. B. Darlington, N. Nicastro, Developmental structure in brain evolution. Behav. Brain Sci. 24, 263-278 (2001).

7. W. de Winter, C. E. Oxnard, Evolutionary radiations and convergences in the structural organization of mammalian brains. Nature 409, 710-714 (2001).

8. S. M. Reader, K. N. Laland, Social intelligence, innovation, and enhanced brain size in primates. Proc. Natl. Acad. Sci. U.S.A. 99, 4436-4441 (2002).

9. L. Lefebvre, S. M. Reader, D. Sol, Brains, innovations and evolution in birds and primates. Brain Behav. Evol. 63, 233-246 (2004). 
10. K. Safi, D. K. N. Dechmann, Adaptation of brain regions to habitat complexity: A comparative analysis in bats (Chiroptera). Proc. Biol. Sci. 272, 179-186 (2005).

11. A. Gonzalez-Voyer, S. Winberg, N. Kolm, Brain structure evolution in a basal vertebrate clade: Evidence from phylogenetic comparative analysis of cichlid fishes. BMC Evol. Biol. 9, 238 (2009).

12. A. Gómez-Robles, W. D. Hopkins, C. C. Sherwood, Modular structure facilitates mosaic evolution of the brain in chimpanzees and humans. Nat. Commun. 5, 4469 (2014).

13. A. N. Iwaniuk, K. M. Dean, J. E. Nelson, A mosaic pattern characterizes the evolution of the avian brain. Proc. Biol. Sci. 271 (Suppl. 4), 148-151 (2004).

14. K. V. Sukhum, J. Shen, B. A. Carlson, Extreme enlargement of the cerebellum in a clade of teleost fishes that evolved a novel active sensory system. Curr. Biol. 28, 3857-3863.e3 (2018).

15. J. R. Krebs, D. F. Sherry, S. D. Healy, V. H. Perry, A. L. Vaccarino, Hippocampal specialization of food-storing birds. Proc. Natl. Acad. Sci. U.S.A. 86, 1388-1392 (1989).

16. D. F. Sherry, A. L. Vaccarino, K. Buckenham, R. S. Herz, The hippocampal complex of food-storing birds. Brain Behav. Evol. 34, 308-317 (2004).

17. L. Z. Garamszegi, M. Eens, The evolution of hippocampus volume and brain size in relation to food hoarding in birds. Ecol. Lett. 7, 1216-1224 (2004).

18. L. Z. Garamszegi, M. Eens, Brain space for a learned task: Strong intraspecific evidence for neural correlates of singing behavior in songbirds. Brain Res. Rev. 44, 187-193 (2004).

19. A. Gonda, G. Herczeg, J. Merilä, Population variation in brain size of nine-spined sticklebacks (Pungitius pungitius) - local adaptation or environmentally induced variation? BMCEvol. Biol. 11, 75 (2011).

20. G. K. H. Zupanc, Neurogenesis and neuronal regeneration in the adult fish brain. J. Comp. Physiol. A 192, 649-670 (2006).

21. A. Gonda, K. Välimäki, G. Herczeg, J. Merilä, Brain development and predation: Plastic responses depend on evolutionary history. Biol. Lett. 8, 249-252 (2012).

22. E. M. Kolb, E. L. Rezende, L. Holness, A. Radtke, S. K. Lee, A. Obenaus, T. Garland Jr., Mice selectively bred for high voluntary wheel running have larger midbrains: Support for the mosaic model of brain evolution. J. Exp. Biol. 216, 515-523 (2013).

23. K. Isler, C. P. van Schaik, The expensive brain: A framework for explaining evolutionary changes in brain size. J. Hum. Evol. 57, 392-400 (2009).

24. A. Kotrschal, B. Rogell, A. Bundsen, B. Svensson, S. Zajitschek, I. Brännström, S. Immler A. A. Maklakov, N. Kolm, Artificial selection on relative brain size in the guppy reveals costs and benefits of evolving a larger brain. Curr. Biol. 23, 168-171 (2013).

25. N. V. Markina, N. V. Popova, I. I. Poletaeva, Interstrain differences in the the behavior of mice selected for greater and lesser brain mass. Zh. Vyssh. Nerv. Deiat. Im. I P Pavlova 49, 59-67 (1999).

26. A. Kotrschal, A. Corral-Lopez, M. Amcoff, N. Kolm, A larger brain confers a benefit in a spatial mate search learning task in male guppies. Behav. Ecol. 26, 527-532 (2015).

27. S. D. Buechel, A. Boussard, A. Kotrschal, W. van der Bijl, N. Kolm, Brain size affects performance in a reversal-learning test. Proc. Biol. Sci. 285, 20172031 (2018).

28. O. V. Perepelkina, I. G. Lilp, A. Y. Tarasova, V. A. Golibrodo, I. I. Poletaeva, Changes in cognitive abilities of laboratory mice as a result of artificial selection. Russ. J. Cogn. Sci. 2, 29-35 (2015).

29. O. V. Perepelkina, A. Y. Tarasova, N. A. Ogienko, I. G. Lil'p, I. I. Poletaeva, Brain weight and cognitive abilities of laboratory mice. Biol. Bull. Rev. 10, 91-101 (2020)

30. A. Corral-López, N. I. Bloch, A. Kotrschal, W. van der Bijl, S. D. Buechel, J. E. Mank, N. Kolm, Female brain size affects the assessment of male attractiveness during mate choice. Sci. Adv. 3, 1601990 (2017).

31. A. Corral-López, M. Romensky, A. Kotrschal, S. D. Buechel, N. Kolm, Brain size affects responsiveness in mating behaviour to variation in predation pressure and sex ratio. J. Evol. Biol. 33, 165-177 (2020)

32. A. Kotrschal, S. D. Buechel, S. M. Zala, A. Corral-López, D. J. Penn, N. Kolm, Brain size affects female but not male survival under predation threat. Ecol. Lett. 18, 646-652 (2015).

33. W. van der Bijl, M. Thyselius, A. Kotrschal, N. Kolm, Brain size affects the behavioural response to predators in female guppies (Poecilia reticulata). Proc. Biol. Sci. 282, 20151132 (2015).

34. A. Kotrschal, N. Kolm, D. J. Penn, Selection for brain size impairs innate, but not adaptive immune responses. Proc. Biol. Sci. 283, 20152857 (2016)

35. A. Kotrschal, A. Corral-Lopez, N. Kolm, Large brains, short life: Selection on brain size impacts intrinsic lifespan. Biol. Lett. 15, 20190137 (2019).

36. A. Kotrschal, A. E. Deacon, A. E. Magurran, N. Kolm, Predation pressure shapes brain anatomy in the wild. Evol. Ecol. 31, 619-633 (2017).

37. R. Huber, M. J. van Staaden, L. S. Kaufman, K. F. Liem, Microhabitat use, trophic patterns, and the evolution of brain structure in African Cichlids. Brain Behav. Evol. 50, 167-182 (1997)

38. L. C. Aiello, P. Wheeler, The expensive-tissue hypothesis: The brain and the digestive system in human and primate evolution. Curr. Anthropol. 36, 199-221 (1995).

39. C. J. Logan, S. Avin, N. Boogert, F. R. Cross, A. Currie, S. Jelbert, R. Mares, A. F. Navarrete, $\mathrm{S}$. Shigeno, S. H. Montgomery, Beyond brain size: Uncovering the neural correlates of behavioral and cognitive specialization. Comp. Cogn. Behav. Rev. 13, 55-90 (2018).
40. M. Portavella, J. P. Vargas, B. Torres, C. Salas, The effects of telencephalic pallial lesions on spatial, temporal, and emotional learning in goldfish. Brain Res. Bull. 57, 397-399 (2002).

41. C. Broglio, F. Rodríguez, C. Salas, Spatial cognition and its neural basis in teleost fishes. Fish Fish. 4, 247-255 (2003).

42. C. Broglio, A. Gómez, E. Durán, F. M. Ocana, F. Jiménez-Moya, F. Rodríguez, C. Salas, Hallmarks of a common forebrain vertebrate plan: Specialized pallial areas for spatial, temporal and emotional memory in actinopterygian fish. Brain Res. Bull. 66, 277-281 (2005).

43. J. G. Corbin, S. Nery, G. Fishell, Telencephalic cells take a tangent: Non-radial migration in the mammalian forebrain. Nat. Neurosci. 4, 1177-1182 (2001).

44. F. Guillemot, Cellular and molecular control of neurogenesis in the mammalian telencephalon. Curr. Opin. Cell Biol. 17, 639-647 (2005).

45. A. Houde, Sex, Color, and Mate Choice in Guppies (Princeton Univ. Press, 1997).

46. D. N. Reznick, M. J. Butler IV, H. Rodd, Life-history evolution in guppies. VII. The comparative ecology of high- and low-predation environments. Am. Nat. 157, 126-140 (2001).

47. D. N. Reznick, Life history evolution in guppies (Poecilia reticulata): Guppies as a model for studying the evolutionary biology of aging. Exp. Gerontol. 32, 245-258 (1997).

48. S. Harris, I. W. Ramnarine, H. G. Smith, L. B. Pettersson, Picking personalities apart: Estimating the influence of predation, sex and body size on boldness in the guppy Poecilia reticulata. Oikos 119, 1711-1718 (2010).

49. J. G. Burns, A. Saravanan, F. Helen Rodd, Rearing environment affects the brain size of guppies: Lab-reared guppies have smaller brains than wild-caught guppies. Ethology 115, 122-133 (2009).

50. L. Marhounová, A. Kotrschal, K. Kverková, N. Kolm, P. Němec, Artificial selection on brain size leads to matching changes in overall number of neurons. Evolution 73, 2003-2012 (2019).

51. A. Gonda, G. Herczeg, J. Merilä, Evolutionary ecology of intraspecific brain size variation: A review. Ecol. Evol. 3, 2751-2764 (2013).

52. K. Noreikiene, G. Herczeg, A. Gonda, G. Balázs, A. Husby, J. Merilä, Quantitative genetic analysis of brain size variation in sticklebacks: Support for the mosaic model of brain evolution. Proc. Biol. Sci. 282, 20151008 (2015).

53. R. Hager, L. Lu, G. D. Rosen, R. W. Williams, Genetic architecture supports mosaic brain evolution and independent brain-body size regulation. Nat. Commun. 3, 1079 (2012).

54. S. B. Laughlin, R. R. de Ruyter van Steveninck, J. C. Anderson, The metabolic cost of neural information. Nat. Neurosci. 1, 36-41 (1998).

55. C. J. Charvet, G. F. Striedter, B. L. Finlay, Evo-devo and brain scaling: Candidate developmental mechanisms for variation and constancy in vertebrate brain evolution. Brain Behav. Evol. 78, 248-257 (2011).

56. G. F. Striedter, R. G. Northcutt, Head size constrains forebrain development and evolution in ray-finned fishes. Evol. Dev. 8, 215-222 (2006).

57. J. E. Niven, S. B. Laughlin, Energy limitation as a selective pressure on the evolution of sensory systems. J. Exp. Biol. 211, 1792-1804 (2008).

58. A. Thornton, D. Lukas, Individual variation in cognitive performance: Developmenta and evolutionary perspectives. Philos. Trans. R. Soc. B Biol. Sci. 367, 2773-2783 (2012).

59. W. G. Eberhard, W. T. Wcislo, Grade changes in brain-body allometry: Morphological and behavioural correlates of brain size in miniature spiders, insects and other invertebrates. Adv. In Insect Phys. 40, 155-214 (2011).

60. S. A. H. Batouli, J. N. Trollor, W. Wen, P. S. Sachdev, The heritability of volumes of brain structures and its relationship to age: A review of twin and family studies. Ageing Res. Rev. 13, 1-9 (2014)

61. A. J. Bartley, D. W. Jones, D. R. Weinberger, Genetic variability of human brain size and cortical gyral patterns. Brain 120, 257-269 (1997).

62. J. M. Cheverud, D. Falk, M. Vannier, L. Konigsberg, R. C. Helmkamp, C. Hildebolt, Heritability of brain size and surface features in rhesus macaques (Macaca mulatta). J. Hered. 81, 51-57 (1990).

63. J. Rogers, P. Kochunov, J. Lancaster, W. Shelledy, D. Glahn, J. Blangero, P. Fox, Heritability of brain volume, surface area and shape: An MRI study in an extended pedigree of baboons. Hum. Brain Mapp. 28, 576-583 (2007).

64. J. S. Peper, R. M. Brouwer, D. I. Boomsma, R. S. Kahn, H. E. Hulshoff Pol, Genetic influences on human brain structure: A review of brain imaging studies in twins. Hum. Brain Mapp. 28, 464-473 (2007).

65. E. V. Sullivan, A. Pfefferbaum, G. E. Swan, D. Carmelli, Heritability of hippocampal size in elderly twin men: Equivalent influence from genes and environment. Hippocampus 11, 754-762 (2001).

66. D. C. Airey, H. Castillo-Juarez, G. Casella, E. J. Pollak, T. J. DeVoogd, Variation in the volume of zebra finch song control nuclei is heritable: Developmental and evolutionary implications. Proc. R. Soc. Lond. B Biol. Sci. 267, 2099-2104 (2000).

67. M. Lynch, B. Walsh, Genetics and Analysis of Quantitative Traits (Sinauer Sunderland, 1998), vol. 1 
68. A. Robertson, Inbreeding in artificial selection programmes. Genet. Res. 2, 189-194 (1961)

69. N. Chen, X. Luo, C. Lu, C. Ke, W. You, Effects of artificial selection practices on loss of genetic diversity in the Pacific abalone, Haliotis discus hannai. Aquac. Res. 48 4923-4933 (2017).

70. P. Beldade, K. Koops, P. M. Brakefield, Developmental constraints versus flexibility in morphological evolution. Nature 416, 844-847 (2002).

71. S. W. Hansen, Selection for behavioural traits in farm mink. Appl. Anim. Behav. Sci. 49, 137-148 (1996).

72. T. von Schantz, M. Tufvesson, G. Göransson, M. Grahn, M. Wilhelmson, H. Wittzell, Artificial selection for increased comb size and its effects on other sexual characters and viability in Gallus domesticus (the domestic chicken). Heredity 75, 518-529 (1995)

73. S. Jackson, J. Diamond, Metabolic and digestive responses to artificial selection in chickens. Evolution 50, 1638-1650 (1996).

74. K. E. Yopak, T. J. Lisney, R. B. Darlington, S. P. Collin, J. C. Montgomery, B. L. Finlay, A conserved pattern of brain scaling from sharks to primates. Proc. Natl. Acad. Sci. U.S.A. 107, 12946-12951 (2010).

75. J. Schindelin, I. Arganda-Carreras, E. Frise, V. Kaynig, M. Longair, T. Pietzsch, S. Preibisch, C. Rueden, S. Saalfeld, B. Schmid, J. Y. Tinevez, D. J. White, V. Hartenstein, K. Eliceiri, P. Tomancak, A. Cardona, Fiji: An open-source platform for biological-image analysis. Nat. Methods 9, 676-682 (2012).

76. A. A. Pollen, A. P. Dobberfuhl, J. Scace, M. M. Igulu, S. C. P. Renn, C. A. Shumway, H. A. Hofmann, Environmental complexity and social organization sculpt the brain in Lake Tanganyikan cichlid fish. Brain Behav. Evol. 70, 21-39 (2007).

77. G. E. White, C. Brown, Variation in brain morphology of intertidal gobies: A comparison of methodologies used to quantitatively assess brain volumes in fish. Brain Behav. Evol. 85, 245-256 (2015).

78. J. W. Mink, R. J. Blumenschine, D. B. Adams, Ratio of central nervous system to body metabolism in vertebrates: Its constancy and functional basis. Am. J. Physiol. Regul. Integr. Comp. Physiol. 241, R203-R212 (1981).
79. RStudio Team, RStudio: Integrated Development for R (RStudio Inc., 2015).

80. J. D. Hadfield, MCMC methods for multi-response generalized linear mixed models: The MCMCgImm R package. J. Stat. Softw. 33, 1-22 (2010)

81. D. Roff, Evolution of Life Histories: Theory and Analysis (Springer Science \& Business Media, 1993).

82. D. Bates, M. Maechler, B. Bolker, S. Walker, Ime4: Linear mixed-effects models using Eigen and S4. R package version 1.1-8 (2015).

Acknowledgments: We acknowledge valuable feedback from R. Vega Trejo and D. Mitchell, and we thank A. Boussard, M. Garate-Olaizola, and V. Holub for help with animal caretaking. We also thank four reviewers and the associate editor for extensive and constructive feedback during the reviewing process. Funding: This study was supported by a Swedish Research Council grant 2016-03435 (N.K.) and a Knut and Alice Wallenberg Foundation grant 102 2013.0072 (N.K.). Ethics statement: The experiment was performed in accordance with ethical applications approved by the Stockholm Animal Research Ethical Permit Board (Dnr: 223/15, N8/17, and 17362-2019). Author contributions: Conceptualization: N.K. Methodology: S.F., A.K., and N.K. Investigation: S.F. Data analyses: S.F., M.A., B.R., and W.v.d.B. Visualization: S.F. Animal maintenance and coordination: S.D.B. and M.A. Supervision: N.K. Writing一original draft: S.F. and N.K. Writing—review and editing: S.F., N.K., B.R., A.K., and W.v.d.B. Competing interests: The authors declare that they have no competing interests. Data and materials availability: All data needed to evaluate the conclusions in the paper are present in the paper and/or the Supplementary Materials. Data and R script used to obtain the statistical outcomes and figures in the present study are available in Dryad digital repository (doi:10.5061/dryad. j6q573nfg). Correspondence should be made to S.F. and N.K.

Submitted 17 May 2021

Accepted 22 September 202

Published 10 November 202

$10.1126 /$ sciadv.abj4314 


\section{ScienceAdvances}

\section{Rapid mosaic brain evolution under artificial selection for relative telencephalon size in the guppy (Poecilia reticulata)}

Stephanie FongBjörn RogellMirjam AmcoffAlexander KotrschalWouter van der BijlSéverine D. BuechelNiclas Kolm

Sci. Adv., 7 (46), eabj4314. • DOI: 10.1126/sciadv.abj4314

View the article online

https://www.science.org/doi/10.1126/sciadv.abj4314

Permissions

https://www.science.org/help/reprints-and-permissions 